\title{
Treatment of fistulas in Crohn's disease with infliximab
}

Present DH, Rutgeerts $\mathrm{P}$, Targan S, et al. Infliximab for the treatment of fistulas in patients with Crohn's disease. N Engl f Med 1999;340:1938-405.

\section{Question}

In patients with Crohn's disease and draining fistulas will treatment with infliximab, monoclonal antibody to tumour necrosis factor $\alpha$, result in fistula closure?

\section{Design}

Randomised, double blind, controlled trial with 18 weeks' follow up.

\section{Setting}

12 centres in the United States $(n=7)$, the UK $(n=2)$, the Netherlands $(n=2)$, and Belgium $(n=1)$.

\section{Patients}

94 Crohn's disease patients aged 18-65 years with single or multiple fistulas ( $90 \%$ perianal, $55 \%$ multiple) were randomised. Patients on stable doses of azathioprine/mercaptopurine $(40 \%)$, corticosteroids $(35 \%)$ and aminosalicylates (55\%) were included but patients on cyclosporin or with other complications such as abscesses or strictures were excluded.

\section{Intervention}

Patients were allocated to either infliximab $5 \mathrm{mg} / \mathrm{kg}$ $(n=31)$, infliximab $10 \mathrm{mg} / \mathrm{kg}(\mathrm{n}=32)$ or placebo $(n=31)$ given as a two hour intravenous infusion at weeks 0,2 and 6.

\section{Main outcome measures}

The primary end point was a $50 \%$ or greater reduction in the number of draining fistulas at two or more consecutive assessments made at weeks $2,6,10,14$, and 18 . Closure of all fistulas, time to response and duration of response were secondary end points.

\section{Main results}

$68 \%$ and $56 \%$ of patients on infliximab $5 \mathrm{mg} / \mathrm{kg}$ and 10 $\mathrm{mg} / \mathrm{kg}$, respectively, achieved the primary end point compared with $26 \%$ of placebo treated patients. Closure of all fistulas occurred in $46 \%$ of infliximab recipients $(55 \%$ on $5 \mathrm{mg} / \mathrm{kg}, 38 \%$ on $10 \mathrm{mg} / \mathrm{kg}$ ) compared with $13 \%$ of placebo recipients. In those responding the median time to response on infliximab $(n=39)$ was 14 days compared with 42 days for the patients responding on placebo $(n=8)$ and the median duration of response (86 days) was the same on infliximab and on placebo. In subgroup analyses infliximab was effective whether or not patients were on corticosteroids or antibiotics but was less effective in patients already on azathioprine or mercaptopurine. Minor adverse events were common in all groups but 5 patients (all on infliximab) suffered adverse events categorised as serious; all 4 occurring on infliximab $10 \mathrm{mg} / \mathrm{kg}$ involved infection.

\section{Comment}

The study performed by Present and coworkers is by far the largest randomised controlled trial (RCT) assessing the use of medical treatment for fistulas in Crohn's disease. Bearing in mind that no other drug has been proved to be efficacious in healing this debilitating condition, a complete response rate (i.e., longstanding closure of all fistulas) of $46 \%$ among the infliximab treated patients compared with $13 \%$ for placebo is impressive. Furthermore, the overall response rate was more than $60 \%$ and lasted for around three months. As in the previously published RCT of infliximab for chronic active Crohn's disease using a single intravenous infusion, ${ }^{1}$ the best response was attained with the lowest dose tested (5 $\mathrm{mg} / \mathrm{kg}$ body weight). In Present et al's study repeated doses of infliximab were given at two and six weeks, but any response occurred before the second infusion had been given in at least half of the patients. In retrospect, one may speculate that a single infusion using the lowest dose would have been enough for a satisfactory response in many patients, but this option was not evaluated. Moreover, the repeated use of the higher $(10 \mathrm{mg} / \mathrm{kg})$ dose was less effective but gave rise to a higher frequency of severe adverse events (pneumonia, abscesses).

Although the report is supplemented by two convincing case studies depicting treatment success in abdominal wall and perianal fistulas, it is difficult to assess fully the severity of the fistulas. Using a composed score for fistula severity ranging from 0 to 20 (highest score for severe activity) the mean score was 9 in both the infliximab and placebo groups, indicating moderate disease. The presence of abscesses was an exclusion criterion, but the use of setons is not mentioned. Despite having had draining fistula(s) for at least three months, only $40 \%$ of the patients had previously used or were current users of azathioprine/6mercaptopurine and less than $30 \%$ were receiving concomitant treatment with an antibiotic. It is not clear whether standard therapy was optimised before inclusion (i.e., higher dose of azathioprine, dual antibiotics, etc.). Interestingly, the group of patients currently on immunosuppressants did not benefit significantly from treatment with infliximab when compared with placebo: $59 v 44 \%$ $(p=0.46)$. However, the overall response to infliximab seemed to be independent of current use of steroids, antibiotics or immunosuppressants.

Formation of human antichimeric antibodies against infliximab took place in just three patients $(5 \%)$ which is fewer than reported in earlier studies. Even though none of the patients had symptoms suggestive of lupus erythematosus, $13 \%$ developed antibodies against double stranded DNA. Long term follow up after monoclonal antibody therapy directed against tumour necrosis factor $\alpha$ may still be warranted but, reassuringly, in a recent survey of 880 patients, mostly with rheumatoid arthritis, ${ }^{2}$ no drug induced lupus erythematosus was seen in patients who had been treated with low doses of infliximab $(3 \mathrm{mg} / \mathrm{kg}$ every eight weeks) over longer periods of up to one year. 


\section{Conclusion}

Treatment with infliximab results in rapid fistula closure, particularly in patients not already taking azathioprine or mercaptopurine.

The promising results from this trial notwithstanding, there are several questions still to be answered before this apparently very potent anti-inflammatory drug may be used as standard therapy for fistulising Crohn's disease: What is the minimum dose of infliximab required for fistula closure and long term healing? Are repeated doses necessary? What is the relapse rate after initial success? Should infliximab be combined with immunosuppressants or antibiotics, or both?

At this stage we know little about the actual healing process induced by infliximab. Is it just the cutaneous orifice of the fistula that heals or the entire fistula tract? What is the risk of abscess formation when the fistula outlet closes? Magnetic resonance imaging has been used to follow the healing process during treatment with infliximab but results from those investigations are not available yet.

Formal RCTs and clinical studies investigating the aforementioned and other questions are essential, particularly in the light of the substantial costs currently associated with the use of a chimeric/humanised monoclonal antibody like infliximab. Finally, the well proved, conservative joint medicosurgical approach to fistulising Crohn's disease should not be abandoned but rather re-evaluated in a controlled fashion in comparison with this new biological alternative.

R LÖFBERG

Department of Gastroenterology, Karolinska Institute, Huddinge University Hospital, Stockholm, Sweden

1 Targan SR, Hanauer SB, van Deventer SJH, et al. A short-term study of chimeric monoclonal antibody cA2 to tumor necrosis factor alpha for Crohn's meric monoclonal antibody cA2 to tumor nec
disease. N Eng f Med 1997;337:1029-35.

2 Smolen JS, Steiner G, Breedveld FC, et al. Anti-TNF $\alpha$ therapy and drug induced lupus-like syndrome [abstract]. XIV EULAR Congress 1999 Abstract Book. London: BMJ Publishing Group, 1999: p217, A906. 